\title{
MODULES WITH RESPECT TO CYCLIC PURITY
}

\author{
LIXIN MAO*
}

\begin{abstract}
An exact sequence $0 \rightarrow A \rightarrow B \rightarrow C \rightarrow 0$ of left $R$-modules is called cyclically pure if for every right ideal $I$ of $R$, the sequence $0 \rightarrow(R / I) \otimes A \rightarrow(R / I) \otimes B \rightarrow(R / I) \otimes C \rightarrow 0$ is exact. In this paper, we study some special modules with respect to cyclic purity, such as $C P$-projective, $C P$-injective and $C P$-flat modules.
\end{abstract}

\section{Introduction}

The notion of purity has an important role in module theory and model theory since it was presented in the literature [5], [18], [22], [23]. There are several generalizations of the notion of purity. Among them, the notion of cyclic purity has been extensively studied by many authors (see, for example, [3], [7], [8], [13], [17]).

In accordance with the terminology of Hochster in [13], an exact sequence $0 \rightarrow A \rightarrow B \rightarrow C \rightarrow 0$ of left $R$-modules is called cyclically pure if for every (finitely generated) right ideal $I$ of $R$, the sequence $0 \rightarrow(R / I) \otimes A \rightarrow$ $(R / I) \otimes B \rightarrow(R / I) \otimes C \rightarrow 0$ is exact. Obviously every pure exact sequence is cyclically pure. But the converse does not hold in general (see [3, Example 1] or $[15$, p. 158-159]).

As in [7], we use the abbreviation $C P$ for the term "cyclically pure". Recall that a left $R$-module $N$ is $C P$-injective [17], [7] if for every cyclically pure exact sequence $0 \rightarrow A \rightarrow B \rightarrow C \rightarrow 0$ of left $R$-modules, the sequence $0 \rightarrow$ $\operatorname{Hom}(C, N) \rightarrow \operatorname{Hom}(B, N) \rightarrow \operatorname{Hom}(A, N) \rightarrow 0$ is exact. A left $R$-module $M$ is called $C P$-projective [8] if for every cyclically pure exact sequence $0 \rightarrow$ $A \rightarrow B \rightarrow C \rightarrow 0$ of left $R$-modules, the sequence $0 \rightarrow \operatorname{Hom}(M, A) \rightarrow$ $\operatorname{Hom}(M, B) \rightarrow \operatorname{Hom}(M, C) \rightarrow 0$ is exact. Clearly, every $C P$-injective (resp. $C P$-projective) module is pure-injective (resp. pure-projective).

* This research was partially supported by NSFC (No. 11071111, 10771096), NSF of Jiangsu Province of China (No. BK2008365), Jiangsu Six Major Talents Peak Project, Jinagsu 333 Project, Jiangsu Qinglan Project and Research Fund of Nanjing Institute of Technology (No. CKJ2009009). The author would like to thank Professor Bjørn Ian Dundas and the referee for the very helpful comments and suggestions.

Received 11 July 2009, in final form 19 March 2010. 
One reason for the importance of cyclic purity is that for some classes of rings, cyclic purity coincides with purity. Following his investigations on "direct summand conjecture" in [13], Hochster explored the structure of Noetherian rings which are pure in any of their cyclically pure extensions. He proved that a Noetherian ring $R$ is pure in every module in which it is cyclically pure if and only if $R$ has small cofinite irreducibles. In [17], Melkersson provided some characterizations for a finitely generated module $M$ over a Noetherian local ring which is pure in every cyclically pure extension of $M$. In [8], Divaani-Aazar, Esmkhani and Tousi characterized locally valuation rings using the coincidences of cyclic purity and purity. In the present paper, we will study the relation between cyclic purity and purity using a different approach. Namely, we introduce the concept of $C P$-flat modules, which is the cyclic purity-relativization of flat modules. It is interesting to note that every right $R$-module is $C P$-flat if and only if every cyclically pure exact sequence of left $R$-modules is pure. Another important observation is that a right $R$ module $N$ is $C P$-flat if and only if the character module $N^{+}$is $C P$-injective. In [7], Divaani-Aazar, Esmkhani and Tousi investigated several properties of $C P$-injective modules. For example, they proved that every module has a $C P$ injective envelope. In this paper, we will give some further applications of these results. In addition, we also deal with many properties of $C P$-projective modules, which may not be dual to properties of $C P$-injective modules. For instance, $C P$-projective covers need not exist in general although $C P$-projective precovers always exist.

Let us now describe the content of the paper in more details.

In Section 2, we first introduce the concept of $C P$-flat modules. We call a right $R$-module $F C P$-flat if for every cyclically pure exact sequence $0 \rightarrow A \rightarrow$ $B \rightarrow C \rightarrow 0$ of left $R$-modules, the sequence $0 \rightarrow F \otimes A \rightarrow F \otimes B \rightarrow F \otimes$ $C \rightarrow 0$ is exact. Some preliminary properties of $C P$-projective, $C P$-injective and $C P$-flat modules are obtained. We then give several characterizations of cyclic purity and describe singly injective modules and flat modules in terms of $C P$-projective and $C P$-injective modules. Finally we prove that the following are equivalent for a ring $R$ and an integer $n \geq 0$ : (1) $\mathrm{wD}(R) \leq n$. (2) Every $C P$-injective left $R$-module has injective dimension $\leq n$. (3) Every $C P$-flat right $R$-module has flat dimension $\leq n$. As a consequence, we characterize von Neumann regular rings and Prüfer rings using $C P$-projective, $C P$-injective and $C P$-flat modules.

In Section 3, we consider the (pre)covers and (pre)envelopes by some special modules, such as $C P$-projective and $C P$-flat modules. In [7], it is shown that every module has a $C P$-injective envelope. Dually, we get that every module has a $C P$-projective precover and a $C P$-flat cover. Next, using these results, we study when the class of $C P$-injective ( $C P$-projective) modules is closed 
under extensions. For example, we prove that the class of $C P$-injective left $R$-modules is closed under extensions if and only if every cotorsion left $R$ module is $C P$-injective. It is also shown that every flat cotorsion left $R$-module is $C P$-injective if and only if the flat cover of every cotorsion left $R$-module is $C P$-injective if and only if the $C P$-injective envelope of every flat left $R$-module is flat.

Section 4 is devoted to some additional characterizations of $C P$-injective and $C P$-projective modules. For example, we show that $M$ is a $C P$-injective left $R$-module if and only if $M$ is injective relative to every cyclically pure exact sequence $0 \rightarrow A \rightarrow B \rightarrow C \rightarrow 0$ of left $R$-modules with $B C P$-projective. Dually, $M$ is a $C P$-projective left $R$-module if and only if $M$ is projective relative to every cyclically pure exact sequence $0 \rightarrow A \rightarrow B \rightarrow C \rightarrow 0$ of left $R$-modules with $B C P$-injective. For a commutative ring $R$, we prove that $M$ is a $C P$-injective $R$-module if and only if $\operatorname{Hom}(F, M)$ is a $C P$-injective $R$-module for any $C P$-flat $R$-module $F$.

Throughout this paper, $R$ is an associative ring with identity and all modules are unitary. $\mathrm{wD}(R)$ stands for the weak global dimension of $R$. The character module $\operatorname{Hom}_{\mathrm{z}}(M, \mathrm{Q} / \mathrm{Z})$ of $M$ is denoted by $M^{+}$. Given $R$-modules $M$ and $N$, $\operatorname{Hom}(M, N)\left(\operatorname{resp} \operatorname{Ext}^{n}(M, N)\right)$ means $\operatorname{Hom}_{R}(M, N)\left(\operatorname{resp} . \operatorname{Ext}_{R}^{n}(M, N)\right)$, and similarly $M \otimes N\left(\right.$ resp. $\left.\operatorname{Tor}_{n}(M, N)\right)$ denotes $M \otimes_{R} N\left(\operatorname{resp} . \operatorname{Tor}_{n}^{R}(M, N)\right)$ for an integer $n \geq 1$. We use freely the module theory terminology and notation introduced in [11], [12], [15], [21], [24].

\section{Definition and general results}

We begin with the following

Definition 2.1. Let $R$ be a ring. A right $R$-module $F$ is called $C P$-flat if for every cyclically pure exact sequence $0 \rightarrow A \rightarrow B \rightarrow C \rightarrow 0$ of left $R$-modules, the sequence $0 \rightarrow F \otimes A \rightarrow F \otimes B \rightarrow F \otimes C \rightarrow 0$ is exact.

Remark 2.2. (1) By the definition, any cyclic right $R$-module is $C P$-flat.

(2) flat right $R$-modules are clearly $C P$-flat. But the converse is not true in general. For example, $\mathrm{Z}_{2}$ is a $C P$-flat $\mathrm{Z}$-module since $\mathrm{Z}_{2}$ is a cyclic $\mathrm{Z}$-module. But it is not a flat Z-module.

Lemma 2.3. Let $R$ be $a$ ring. Then

(1) A right $R$-module $N$ is $C P$-flat if and only if $N^{+}$is $C P$-injective.

(2) The class of CP-flat right $R$-modules is closed under pure submodules, pure quotient modules and direct limits.

Proof. (1) Let $0 \rightarrow A \rightarrow B \rightarrow C \rightarrow 0$ be a cyclically pure exact sequence of left $R$-modules and $N$ a right $R$-module. Then the sequence $0 \rightarrow$ 
$N \otimes A \rightarrow N \otimes B \rightarrow N \otimes C \rightarrow 0$ is exact if and only if the sequence $0 \rightarrow(N \otimes C)^{+} \rightarrow(N \otimes B)^{+} \rightarrow(N \otimes A)^{+} \rightarrow 0$ is exact if and only if the sequence $0 \rightarrow \operatorname{Hom}\left(C, N^{+}\right) \rightarrow \operatorname{Hom}\left(B, N^{+}\right) \rightarrow \operatorname{Hom}\left(A, N^{+}\right) \rightarrow 0$ is exact. So $N$ is $C P$-flat if and only if $N^{+}$is $C P$-injective.

(2) Let $0 \rightarrow A \rightarrow B \rightarrow C \rightarrow 0$ be a pure exact sequence of right $R$ modules with $B C P$-flat. Then we get the split exact sequence $0 \rightarrow C^{+} \rightarrow$ $B^{+} \rightarrow A^{+} \rightarrow 0$. Since $B^{+}$is $C P$-injective by (1), $A^{+}$and $C^{+}$are $C P$-injective. So $A$ and $C$ are $C P$-flat.

In addition, the class of $C P$-flat right $R$-modules is clearly closed under direct limits.

COROLlary 2.4. The following are equivalent for a ring $R$ :

(1) Every right $R$-module is $C P$-flat.

(2) Every cyclically pure exact sequence $0 \rightarrow A \rightarrow B \rightarrow C \rightarrow 0$ of left $R$-modules is pure.

(3) Every pure-projective left $R$-module is $C P$-projective.

(4) Every pure-injective left $R$-module is $C P$-injective.

Proof. (1) $\Leftrightarrow(2) \Leftrightarrow(3)$ and $(2) \Rightarrow(4)$ are clear.

(4) $\Rightarrow$ (1) Let $M$ be a right $R$-module. Then $M^{+}$is pure-injective and so is $C P$-injective by (4). Thus $M$ is $C P$-flat by Lemma 2.3 (1).

In what follows, $\mathscr{S}$ denotes the set of all left $R$-modules of the form $R^{n} / G$ for all $n \in \mathrm{N}$ and all cyclic submodules $G$ of $R^{n}$.

Next we present further characterizations of cyclically pure exact sequences.

Lemma 2.5. The following are equivalent for an exact sequence $0 \rightarrow A \rightarrow$ $B \rightarrow C \rightarrow 0$ of left $R$-modules:

(1) $0 \rightarrow A \rightarrow B \rightarrow C \rightarrow 0$ is cyclically pure.

(2) The sequence $0 \rightarrow \operatorname{Hom}(M, A) \rightarrow \operatorname{Hom}(M, B) \rightarrow \operatorname{Hom}(M, C) \rightarrow 0$ is exact for any $M \in \mathscr{S}$.

(3) The sequence $0 \rightarrow \operatorname{Hom}(M, A) \rightarrow \operatorname{Hom}(M, B) \rightarrow \operatorname{Hom}(M, C) \rightarrow 0$ is exact for any $C P$-projective left $R$-module $M$.

(4) The sequence $0 \rightarrow \operatorname{Hom}(C, N) \rightarrow \operatorname{Hom}(B, N) \rightarrow \operatorname{Hom}(A, N) \rightarrow 0$ is exact for any $C P$-injective left $R$-module $N$.

(5) Every cyclic right $R$-module is projective relative to the exact sequence $0 \rightarrow C^{+} \rightarrow B^{+} \rightarrow A^{+} \rightarrow 0$.

(6) The sequence $0 \rightarrow C^{+} \otimes M \rightarrow B^{+} \otimes M \rightarrow A^{+} \otimes M \rightarrow 0$ is exact for any $M \in \mathscr{S}$.

(7) The sequence $0 \rightarrow F \otimes A \rightarrow F \otimes B \rightarrow F \otimes C \rightarrow 0$ is exact for any $C P$-flat right $R$-module $F$. 
Proof. (1) $\Leftrightarrow(2)$ holds by [7, Proposition 2.2].

(3) $\Leftrightarrow(1) \Leftrightarrow$ (7) and (1) $\Rightarrow$ (4) are clear.

(4) $\Rightarrow(1)$ Let $I$ be a right ideal of $R$. By Lemma $2.3(1),(R / I)^{+}$is $C P$ injective. Thus by (4), we obtain the exact sequence

$0 \longrightarrow \operatorname{Hom}\left(C,(R / I)^{+}\right) \longrightarrow \operatorname{Hom}\left(B,(R / I)^{+}\right)$

$$
\longrightarrow \operatorname{Hom}\left(A,(R / I)^{+}\right) \longrightarrow 0,
$$

which gives rise to the exact sequence

$$
0 \longrightarrow((R / I) \otimes C)^{+} \longrightarrow((R / I) \otimes B)^{+} \longrightarrow((R / I) \otimes A)^{+} \longrightarrow 0 .
$$

So we get the exact sequence

$$
0 \longrightarrow(R / I) \otimes A \longrightarrow(R / I) \otimes B \longrightarrow(R / I) \otimes C \longrightarrow 0 .
$$

Therefore $0 \rightarrow A \rightarrow B \rightarrow C \rightarrow 0$ is a cyclically pure exact sequence.

$(1) \Leftrightarrow(5)$ Let $I$ be a right ideal of $R$. Then the exact sequence $0 \rightarrow(R / I) \otimes$ $A \rightarrow(R / I) \otimes B \rightarrow(R / I) \otimes C \rightarrow 0$ is exact if and only if $0 \rightarrow((R / I) \otimes$ $C)^{+} \rightarrow((R / I) \otimes B)^{+} \rightarrow((R / I) \otimes A)^{+} \rightarrow 0$ is exact if and only if $0 \rightarrow$ $\operatorname{Hom}\left(R / I, C^{+}\right) \rightarrow \operatorname{Hom}\left(R / I, B^{+}\right) \rightarrow \operatorname{Hom}\left(R / I, A^{+}\right) \rightarrow 0$ is exact. So $(1) \Leftrightarrow(5)$ holds.

(2) $\Leftrightarrow(6)$ Let $M \in \mathscr{S}$. Then we get the following commutative diagram:

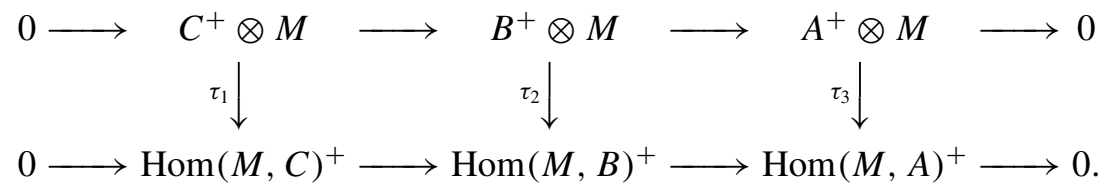

By [6, Lemma 2], $\tau_{1}, \tau_{2}$ and $\tau_{3}$ are isomorphisms. Thus the first row is exact if and only if the second row is exact if and only if the sequence $0 \rightarrow$ $\operatorname{Hom}(M, A) \rightarrow \operatorname{Hom}(M, B) \rightarrow \operatorname{Hom}(M, C) \rightarrow 0$ is exact. So $(2) \Leftrightarrow(6)$ follows.

The next lemma will be used frequently in the sequel.

Lemma 2.6. Let $R$ be a ring. Then

(1) For any left $R$-module $M$, there exists a cyclically pure exact sequence $0 \rightarrow N \rightarrow \bigoplus C_{i} \rightarrow M \rightarrow 0$ with $C_{i} \in \mathscr{S}$.

(2) A left $R$-module $M$ is $C P$-projective if and only if $M$ is a direct summand of $\bigoplus C_{i}$ with $C_{i} \in \mathscr{S}$. 
Proof. (1) Let $M$ be a left $R$-module. Given any $G \in \mathscr{S}$, there is the evaluation map

$$
\bigoplus_{\operatorname{Hom}(G, M)} G \longrightarrow M
$$

So we get the induced map

$$
\bigoplus_{G \in \mathscr{S}} \bigoplus_{\operatorname{Hom}(G, M)} G \stackrel{\alpha}{\longrightarrow} M
$$

Thus we get the exact sequence

$$
\operatorname{Hom}\left(G, \bigoplus_{\operatorname{Hom}(G, M)} G\right) \longrightarrow \operatorname{Hom}(G, M) \longrightarrow 0
$$

Therefore for any $G^{\prime} \in \mathscr{S}$, we have the exact sequence

$$
\operatorname{Hom}\left(G^{\prime}, \bigoplus_{G \in \mathscr{S}} \bigoplus_{\operatorname{Hom}(G, M)} G\right) \stackrel{\alpha_{*}}{\longrightarrow} \operatorname{Hom}\left(G^{\prime}, M\right) \longrightarrow 0
$$

Since $R \in \mathscr{S}, \alpha$ is epic. So by Lemma 2.5 , we have the cyclically pure exact sequence

$$
0 \longrightarrow K \longrightarrow \bigoplus_{G \in \mathscr{S}} \bigoplus_{\operatorname{Hom}(G, M)} G \stackrel{\alpha}{\longrightarrow} M \longrightarrow 0
$$

(2) is easy by (1).

Following [16], a left $R$-module $M$ is said to be singly injective if $\operatorname{Ext}^{1}(G$, $M)=0$ for any $G \in \mathscr{S}$. A right $R$-module $N$ is called singly flat if $\operatorname{Tor}_{1}(N$, $G)=0$ for any $G \in \mathscr{S}$. By [16, Lemma 2.4], a right $R$-module $N$ is singly flat if and only if $N^{+}$is singly injective. There exist close connections between singly injectivity and cyclic purity as shown by the following proposition.

Proposition 2.7. The following are equivalent for a left $R$-module $M$ :

(1) $M$ is singly injective.

(2) $\operatorname{Ext}^{1}(N, M)=0$ for any $C P$-projective left $R$-module $N$.

(3) For every $C P$-injective left $R$-module $G$, every homomorphism $M \rightarrow G$ factors through an injective left $R$-module.

(4) Every exact sequence $0 \rightarrow M \rightarrow B \rightarrow C \rightarrow 0$ is cyclically pure.

(5) There exists a cyclically pure exact sequence $0 \rightarrow M \rightarrow E \rightarrow F \rightarrow 0$ with E singly injective.

Proof. (1) $\Rightarrow$ (2) follows from Lemma 2.6 (2). 
(2) $\Rightarrow$ (3) There is an exact sequence $0 \rightarrow M \rightarrow E \rightarrow C \rightarrow 0$ with $E$ injective. By (2), for any $A \in \mathscr{S}$, we have the exact sequence

$$
0 \rightarrow \operatorname{Hom}(A, M) \rightarrow \operatorname{Hom}(A, E) \rightarrow \operatorname{Hom}(A, C) \rightarrow \operatorname{Ext}^{1}(A, M)=0 .
$$

Thus $0 \rightarrow M \rightarrow E \rightarrow C \rightarrow 0$ is cyclically pure, and so every homomorphism $M \rightarrow G$ with $G C P$-injective factors through $E$.

(3) $\Rightarrow$ (4) Let $0 \rightarrow M \stackrel{i}{\rightarrow} B \rightarrow C \rightarrow 0$ be an exact sequence. For any $C P$-injective left $R$-module $G$ and any homomorphism $f: M \rightarrow G$, there are an injective left $R$-module $E$ and $g: M \rightarrow E$ and $h: E \rightarrow G$ such that $f=h g$ by (3). Since $\mathrm{E}$ is injective, there is $\alpha: B \rightarrow E$ such that $\alpha i=g$. Thus $f=(h \alpha) i$. So the sequence $0 \rightarrow M \stackrel{i}{\rightarrow} B \rightarrow C \rightarrow 0$ is cyclically pure by Lemma 2.5 .

(4) $\Rightarrow(5)$ is easy since $M$ embeds in an injective $R$-module.

(5) $\Rightarrow$ (1) Let $0 \rightarrow M \rightarrow E \rightarrow F \rightarrow 0$ be a cyclically pure exact sequence with $E$ singly injective. For any $N \in \mathscr{S}$, we have the induced exact sequence

$$
\operatorname{Hom}(N, E) \longrightarrow \operatorname{Hom}(N, F) \longrightarrow \operatorname{Ext}^{1}(N, M) \longrightarrow \operatorname{Ext}^{1}(N, E)=0 .
$$

Since $\operatorname{Hom}(N, E) \rightarrow \operatorname{Hom}(N, F) \rightarrow 0$ is exact, $\operatorname{Ext}^{1}(N, M)=0$, and so $M$ is singly injective.

Recall that a ring $R$ is left $P P$ if every principal left ideal of $R$ is projective.

COROLlaRY 2.8. The following are equivalent for a ring $R$ :

(1) $R$ is a left $P P$ ring.

(2) Every quotient module of a singly injective left $R$-module is singly injective.

(3) Every CP-projective left $R$-module has projective dimension $\leq 1$.

PRoOF. (1) $\Leftrightarrow$ (2) holds by [16, Theorem 3.2].

(2) $\Rightarrow$ (3) Let $M$ be a $C P$-projective left $R$-module and $N$ any left $R$-module. Then there is an exact sequence $0 \rightarrow N \rightarrow E \rightarrow L \rightarrow 0$ with $E$ injective, which induces the exact sequence

$$
0=\operatorname{Ext}^{1}(M, E) \longrightarrow \operatorname{Ext}^{1}(M, L) \longrightarrow \operatorname{Ext}^{2}(M, N) \longrightarrow \operatorname{Ext}^{2}(M, E)=0 .
$$

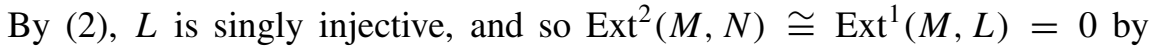
Proposition 2.7. It follows that $M$ has projective dimension $\leq 1$.

(3) $\Rightarrow$ (1) Let $I$ be a principal left ideal of $R$. Since $R / I$ has projective dimension $\leq 1$ by (3), $I$ is projective. So $R$ is a left $P P$ ring.

It is well known that a left $R$-module $N$ is flat if and only if every homomorphism $G \rightarrow N$ with $G$ any finitely presented left $R$-module factors 
through a projective left $R$-module if and only if every exact sequence $0 \rightarrow$ $K \rightarrow Q \rightarrow N \rightarrow 0$ is pure. The following theorem gives some interesting characterizations of flat modules in terms of cyclic purity.

THEOREM 2.9. The following are equivalent for a left $R$-module $N$ :

(1) $N$ is flat.

(2) $\operatorname{Ext}^{1}(N, M)=0$ for any $C P$-injective left $R$-module $M$.

(3) For every $C P$-projective left $R$-module $G$, every homomorphism $G \rightarrow N$ factors through a projective left $R$-module.

(4) Every exact sequence $0 \rightarrow K \rightarrow Q \rightarrow N \rightarrow 0$ is cyclically pure.

(5) There exists a cyclically pure exact sequence $0 \rightarrow M \rightarrow F \rightarrow N \rightarrow 0$ with $F$ flat.

Proof. (1) $\Rightarrow$ (2) There is an exact sequence $0 \rightarrow K \rightarrow P \rightarrow N \rightarrow 0$ with $P$ projective. Since $N$ is flat, the exact sequence $0 \rightarrow K \rightarrow P \rightarrow N \rightarrow 0$ is (cyclically) pure. Thus $\operatorname{Hom}(P, M) \rightarrow \operatorname{Hom}(K, M) \rightarrow 0$ is exact for any $C P$-injective left $R$-module $M$. Consider the induced exact sequence

$$
\operatorname{Hom}(P, M) \longrightarrow \operatorname{Hom}(K, M) \longrightarrow \operatorname{Ext}^{1}(N, M) \longrightarrow \operatorname{Ext}^{1}(P, M)=0 .
$$

So $\operatorname{Ext}^{1}(N, M)=0$.

(2) $\Rightarrow$ (3) There is an exact sequence $0 \rightarrow K \rightarrow P \rightarrow N \rightarrow 0$ with $P$ projective. For any $C P$-injective left $R$-module $M$, by (2), we have the exact sequence

$$
0 \rightarrow \operatorname{Hom}(N, M) \rightarrow \operatorname{Hom}(P, M) \rightarrow \operatorname{Hom}(K, M) \rightarrow \operatorname{Ext}^{1}(N, M)=0 .
$$

Thus $0 \rightarrow K \rightarrow P \rightarrow N \rightarrow 0$ is cyclically pure by Lemma 2.5 , and so every homomorphism $G \rightarrow N$ with $G C P$-projective factors through $P$.

(3) $\Rightarrow$ (4) Let $0 \rightarrow K \rightarrow Q \stackrel{\pi}{\rightarrow} N \rightarrow 0$ be an exact sequence. For any $C P$-projective left $R$-module $G$ and any homomorphism $f: G \rightarrow N$, there exist a projective left $R$-module $P$ and $g: G \rightarrow P$ and $h: P \rightarrow N$ such that $f=h g$ by (3). Since $\mathrm{P}$ is projective, there is $\alpha: P \rightarrow Q$ such that $\pi \alpha=h$. Thus $f=\pi(\alpha g)$. So the sequence $0 \rightarrow K \rightarrow Q \stackrel{\pi}{\rightarrow} N \rightarrow 0$ is cyclically pure by Lemma 2.5 .

(4) $\Rightarrow(5)$ There exists an exact sequence $0 \rightarrow K \rightarrow P \rightarrow N \rightarrow 0$ with $P$ projective, which is cyclically pure by (4).

(5) $\Rightarrow$ (1) Let $0 \rightarrow M \rightarrow F \rightarrow N \rightarrow 0$ be a cyclically pure exact sequence with $F$ flat. For any right ideal $I$, we have the exact sequence

$$
0=\operatorname{Tor}_{1}(R / I, F) \longrightarrow \operatorname{Tor}_{1}(R / I, N) \longrightarrow(R / I) \otimes M \longrightarrow(R / I) \otimes F .
$$


Since $(R / I) \otimes M \rightarrow(R / I) \otimes F$ is monic, $\operatorname{Tor}_{1}(R / I, N)=0$, and so $N$ is flat.

As an immediate consequence of Theorem 2.9, we get

Corollary 2.10. Let $0 \rightarrow A \rightarrow B \rightarrow C \rightarrow 0$ be a cyclically pure exact sequence of left $R$-modules with $B$ projective and A finitely generated. Then $A$ is projective.

Proof. Since $A$ is finitely generated and $B$ is projective, without loss of generality, we may assume that $B$ is finitely generated. So $C$ is finitely presented. By Theorem 2.9, $C$ is flat. Thus $C$ is projective. Hence $A$ is isomorphic to a direct summand of $B$, and so is projective.

The following corollary clarifies the relationship between $C P$-injective (resp. $C P$-projective, $C P$-flat) modules and injective (resp. projective, flat) modules.

COROLlary 2.11. The following are true for any ring $R$ :

(1) Any singly injective $C P$-injective left $R$-module is injective.

(2) Any flat CP-projective left $R$-module is projective.

(3) Any singly flat $C P$-flat right $R$-module is flat.

Proof. (1) Let $M$ be any singly injective $C P$-injective left $R$-module. By Proposition 2.7, there exists a cyclically pure exact sequence $0 \rightarrow M \rightarrow$ $E \rightarrow C \rightarrow 0$ with $E$ injective. So the exact sequence is split, and hence $M$ is injective.

(2) Let $N$ be any flat $C P$-projective left $R$-module. By Theorem 2.9, there is a cyclically pure exact sequence $0 \rightarrow K \rightarrow P \rightarrow N \rightarrow 0$ with $P$ projective. Thus the exact sequence is split, and hence $N$ is projective.

(3) Let $G$ be any singly flat $C P$-flat right $R$-module. Then $G^{+}$is singly injective $C P$-injective, and so is injective by (1). Thus $G$ is flat.

Recall that a left $R$-module $C$ is cotorsion [10] if $\operatorname{Ext}^{1}(F, C)=0$ for every flat left $R$-module $F$. By Theorem 2.9, any $C P$-injective left $R$-module is cotorsion. But the converse is not true in general (see [25, p. 75, Example]).

The equivalence of (1) and (2) in the following theorem has been proved by $\mathrm{Xu}$ (see [25, Theorem 3.3.2]). But here we give an easy proof.

THEOREM 2.12. The following are equivalent for a ring $R$ and an integer $n \geq 0$ :

(1) $\mathrm{wD}(R) \leq n$.

(2) Every cotorsion left $R$-module has injective dimension $\leq n$. 
(3) Every CP-injective left $R$-module has injective dimension $\leq n$.

(4) Every $C P$-flat right $R$-module has flat dimension $\leq n$.

Proof. (1) $\Rightarrow(2)$ Let $M$ be a cotorsion left $R$-module and $N$ any left $R$ module. Then there is an exact sequence

$$
0 \longrightarrow K_{n} \longrightarrow P_{n-1} \longrightarrow \cdots \longrightarrow P_{1} \longrightarrow P_{0} \longrightarrow N \longrightarrow 0
$$

with each $P_{i}$ projective. By (1), $K_{n}$ is flat, and so

$$
\operatorname{Ext}^{n+1}(N, M) \cong \operatorname{Ext}^{1}\left(K_{n}, M\right)=0 .
$$

It follows that $M$ has injective dimension $\leq n$.

(2) $\Rightarrow(3)$ is trivial.

(3) $\Rightarrow$ (4) For any $C P$-flat right $R$-module $A, A^{+}$is $C P$-injective. By (3), for every left $R$-module $B$, we have

$$
\operatorname{Tor}_{n+1}(A, B)^{+} \cong \operatorname{Ext}^{n+1}\left(B, A^{+}\right)=0 .
$$

So $\operatorname{Tor}_{n+1}(A, B)=0$, and hence $A$ has flat dimension $\leq n$.

(4) $\Rightarrow$ (1) is clear since every cyclic right $R$-module is $C P$-flat.

As a consequence of Theorem 2.12, we obtain new characterizations of von Neumann regular rings and Prüfer rings as follows.

COROLLARY 2.13. The following are equivalent for a ring $R$ :

(1) $R$ is a von Neumann regular ring.

(2) Every $C P$-injective left $R$-module is injective.

(3) Every $C P$-flat right $R$-module is flat.

(4) Every $C P$-projective left $R$-module is projective.

(5) Every exact sequence $0 \rightarrow A \rightarrow B \rightarrow C \rightarrow 0$ of left $R$-modules is cyclically pure.

Proof. (1) $\Leftrightarrow(2) \Leftrightarrow$ (3) follow from Theorem 2.12.

$(1) \Rightarrow(4)$ is easy by Lemma 2.6 (2).

(4) $\Rightarrow$ (1) Let $I$ be a principal left ideal of $R$. Then $R / I$ is projective by (4), and so $I$ is a direct summand of $R$. Thus $R$ is a von Neumann regular ring.

(1) $\Leftrightarrow(5)$ holds by Theorem 2.9.

COROLLARY 2.14. The following are equivalent for a commutative domain $R$ :

(1) $R$ is a Prüfer ring.

(2) Every $C P$-injective $R$-module has injective dimension $\leq 1$. 
(3) Every $C P$-flat $R$-module has flat dimension $\leq 1$.

(4) Every $R$-module is CP-flat.

Proof. It is known that a commutative domain $R$ is a Prüfer ring if and only if every ideal of $R$ is flat if and only if $\mathrm{wD}(R) \leq 1$ (see [24, 40.4]). So (1) $\Leftrightarrow(2) \Leftrightarrow$ (3) follow from Theorem 2.12.

$(1) \Leftrightarrow(4)$ holds by Corollary 2.4 and [8, Corollary 2.11].

\section{Some (pre)covers and (pre)envelopes}

Let $\mathscr{C}$ be a class of $R$-modules and $M$ an $R$-module. Recall that a homomorphism $\phi: C \rightarrow M$ is a $\mathscr{C}$-precover of $M$ [9] if $C \in \mathscr{C}$ and the abelian group homomorphism $\operatorname{Hom}\left(C^{\prime}, \phi\right): \operatorname{Hom}\left(C^{\prime}, C\right) \rightarrow \operatorname{Hom}\left(C^{\prime}, M\right)$ is surjective for every $C^{\prime} \in \mathscr{C}$. A $\mathscr{C}$-precover $\phi: C \rightarrow M$ is said to be a $\mathscr{C}$-cover of $M$ if every endomorphism $g: C \rightarrow C$ such that $\phi g=\phi$ is an isomorphism. Dually we have the definitions of a $\mathscr{C}$-preenvelope and a $\mathscr{C}$-envelope. $\mathscr{C}$-covers $(\mathscr{C}$-envelopes) may not exist in general, but if they exist, they are unique up to isomorphism. When specializing $\mathscr{C}$ to the class of injective modules and projective modules respectively, $\mathscr{C}$-envelopes and $\mathscr{C}$-covers agree with the usual injective envelopes and projective covers respectively (see [25]).

In this section, we first investigate the existence of (pre)covers and (pre)envelopes by modules with respect to cyclic purity.

Recall that $R$ is a left coherent ring [4] if every finitely generated left ideal of $R$ is finitely presented.

Proposition 3.1. Let $R$ be a left coherent ring. Then

(1) Every CP-projective right $R$-module has a projective preenvelope.

(2) Every $C P$-injective left $R$-module has an injective cover.

Proof. (1) If $M$ is a $C P$-projective right $R$-module, then by [9, Proposition 5.1], $M$ has a flat preenvelope $f: M \rightarrow F$. By Theorem 2.9, $f$ factors through a projective right $R$-module $P$, i.e., there exist $g: M \rightarrow P$ and $h: P \rightarrow F$ such that $f=h g$. It is easy to see that $g$ is a projective preenvelope of $M$.

(2) Let $M$ be a $C P$-injective left $R$-module. By [16, Theorem 2.15], $M$ has a singly injective cover $f: F \rightarrow M$. There is an exact sequence $0 \rightarrow F \stackrel{i}{\rightarrow}$ $E \rightarrow L \rightarrow 0$ with $E$ injective. Since the exact sequence is cyclically pure by Proposition 2.7, there exists $g: E \rightarrow M$ such that $g i=f$. So there exists $\varphi: E \rightarrow F$ such that $f \varphi=g$ since $f$ is a singly injective cover. Therefore $f \varphi i=f$ and hence $\varphi i$ is an isomorphism. It follows that $F$ is isomorphic to a direct summand of $E$, and so $F$ is injective. Thus $f$ is an injective cover of $M$. 
By [7, Theorem 4.10], every $R$-module $M$ has a $C P$-injective envelope $i: M \rightarrow N$. Moreover, the exact sequence $0 \rightarrow M \stackrel{i}{\rightarrow} N \rightarrow L \rightarrow 0$ is cyclically pure.

THEOREM 3.2. Let $R$ be a ring. Then

(1) Every left $R$-module has a CP-projective precover.

(2) Every left $R$-module has a CP-projective cover if and only if the class of $C P$-projective left $R$-modules is closed under direct limits if and only if the class of $C P$-projective left $R$-modules is closed under pure quotient modules.

(3) Every right $R$-module has a $C P$-flat cover.

(4) Every right $R$-module has a $C P$-flat preenvelope if and only if the class of $C P$-flat right $R$-modules is closed under direct products.

Proof. (1) is clear by Lemma 2.6 (1).

(2) is easy by [1, Theorem 2.13].

(3) follows from [14, Theorem 2.5] and Lemma 2.3 (2).

(4) holds by [19, Corollary 3.5 (c)] and Lemma 2.3 (2).

REMARK 3.3. Although $C P$-projective precovers always exist, $C P$-projective covers need not exist in general. In fact, the ring $Z$ is hereditary but not pure semisimple. By [8, Corollary 2.11], CP-projective Z-modules coincide with pure projective Z-modules. So not every Z-module has a $C P$-projective cover by [1, Corollary 6.18].

Now we study when the class of $C P$-projective ( $C P$-injective) left $R$-modules is closed under extensions.

We will call a left $R$-module $M \mathscr{S}$-projective if $\operatorname{Ext}^{1}(M, G)=0$ for any singly injective left $R$-module $G$. Obviously, any $C P$-projective left $R$-module is $\mathscr{S}$-projective by Proposition 2.7 .

By [12, Corollary 3.2.4], $M$ is $\mathscr{S}$-projective if and only if $M$ is a direct summand in a left $R$-module $N$ such that $N$ is a union of a continuous chain, $\left(N_{\alpha}: \alpha<\lambda\right)$, for a cardinal $\lambda, N_{0}=0$ and $N_{\alpha+1} / N_{\alpha}$ is isomorphic to a left $R$-module in $\mathscr{S}$ for all $\alpha<\lambda$.

Proposition 3.4. If the class of CP-projective left $R$-modules is closed under direct limits, then the following are equivalent:

(1) The class of $C P$-projective left $R$-modules is closed under extensions.

(2) Every $\mathscr{S}$-projective left $R$-module is $C P$-projective.

Proof. (1) $\Rightarrow(2)$ Let $M$ be an $\mathscr{S}$-projective left $R$-module. By Theorem 3.2 (2), we have an exact sequence $0 \rightarrow K \rightarrow C \rightarrow M \rightarrow 0$, where 
$C \rightarrow M$ is a $C P$-projective cover of $M$. By (1) and Wakamatsu's Lemma (see [25, Lemma 2.1.1]), $\operatorname{Ext}^{1}(N, K)=0$ for every $C P$-projective left $R$-module $N$, and so $K$ is singly injective by Proposition 2.7. Therefore $\operatorname{Ext}^{1}(M, K)=0$, and hence the sequence $0 \rightarrow K \rightarrow C \rightarrow M \rightarrow 0$ is split. Thus $M$ is isomorphic to a direct summand of $C$, and so is $C P$-projective.

$(2) \Rightarrow(1)$ is obvious.

Dually, we have

Proposition 3.5. The following are equivalent for a ring $R$ :

(1) The class of CP-injective left $R$-modules is closed under extensions.

(2) Every cotorsion left $R$-module is $C P$-injective.

In this case, the class of CP-flat right $R$-modules is also closed under extensions.

Proof. (1) $\Rightarrow(2)$ Let $M$ be a cotorsion left $R$-module. By [7, Theorem 4.10], we have an exact sequence $0 \rightarrow M \rightarrow N \rightarrow L \rightarrow 0$, where $M \rightarrow N$ is a $C P$-injective envelope of $M$. By (1) and Wakamatsu's Lemma (see [25, Lemma 2.1.2]), $\operatorname{Ext}^{1}(L, C)=0$ for every $C P$-injective left $R$-module $C$, and so $L$ is flat by Theorem 2.9. Therefore $\operatorname{Ext}^{1}(L, M)=0$, and hence the sequence $0 \rightarrow M \rightarrow N \rightarrow L \rightarrow 0$ is split. Thus $M$ is isomorphic to a direct summand of $N$ and so is $C P$-injective.

(2) $\Rightarrow(1)$ is obvious.

In this case, if $0 \rightarrow A \rightarrow B \rightarrow C \rightarrow 0$ is an exact sequence of right $R$-modules with $A$ and $C C P$-flat, then we get the exact sequence $0 \rightarrow C^{+} \rightarrow$ $B^{+} \rightarrow A^{+} \rightarrow 0$. By Lemma $2.3(1), A^{+}$and $C^{+}$are $C P$-injective. Thus $B^{+}$ is $C P$-injective, and hence $B$ is $C P$-flat.

It is well known that all $R$-modules have flat covers for any ring $R$ [2]. Since every $R$-module has a cotorsion envelope if and only if every $R$-module has a flat cover [25], all $R$-modules have cotorsion envelopes for an arbitrary ring $R$.

By Wakamatsu's Lemma, the cotorsion envelope of every flat $R$-module is flat. In [20], Rothmaler considered when the cotorsion envelope of every flat $R$-module is pure-injective. Motivated by this idea, we next study when the cotorsion envelope of every flat $R$-module is $C P$-injective.

THEOREM 3.6. The following are equivalent for a ring $R$ :

(1) Every flat cotorsion left $R$-module is $C P$-injective.

(2) If $0 \rightarrow A \rightarrow B \rightarrow C \rightarrow 0$ is an exact sequence of left $R$-modules, where $A$ is $C P$-injective and $C$ is a $C P$-injective envelope of a flat left $R$-module, then $B$ is $C P$-injective. 
(3) The flat cover of every cotorsion left $R$-module is $C P$-injective.

(4) The flat cover of every $C P$-injective left $R$-module is $C P$-injective.

(5) The CP-injective envelope of every flat left $R$-module is flat.

(6) The cotorsion envelope of every flat left $R$-module is $C P$-injective.

Proof. (1) $\Rightarrow(3)$ is easy since the flat cover of every cotorsion left $R$ module is cotorsion by Wakamatsu's Lemma.

(3) $\Rightarrow$ (4) is trivial.

(4) $\Rightarrow$ (5) Let $M$ be a flat left $R$-module, $\lambda: M \rightarrow N$ the $C P$-injective envelope, and $\mu: F \rightarrow N$ the flat cover of $N$. Then there exists $\alpha: M \rightarrow F$ such that $\mu \alpha=\lambda$. On the other hand, since $F$ is $C P$-injective by (4), there exists $\gamma: N \rightarrow F$ such that $\gamma \lambda=\alpha$. Thus $(\mu \gamma) \lambda=\lambda$, and so $\mu \gamma$ is an isomorphism since $\lambda$ is an envelope. It follows that $N$ is flat.

(5) $\Rightarrow$ (1) Let $M$ be a flat cotorsion left $R$-module. By [7, Theorem 4.10], we have an exact sequence $0 \rightarrow M \rightarrow N \rightarrow L \rightarrow 0$, where $M \rightarrow N$ is a $C P$-injective envelope of $M$, and the sequence is cyclically pure. By (5), $N$ is flat, and so $L$ is flat by Theorem 2.9. Therefore $\operatorname{Ext}^{1}(L, M)=0$, and hence the sequence $0 \rightarrow M \rightarrow N \rightarrow L \rightarrow 0$ is split. Thus $M$ is $C P$-injective.

(2) $\Rightarrow$ (5) Let $N$ be the $C P$-injective envelope of a flat left $R$-module $M$ and $\lambda: M \rightarrow N$ be the inclusion map. We will first show that $\operatorname{Ext}^{1}(N / M, K)=0$ for any $C P$-injective left $R$-module $K$. In fact, let $0 \rightarrow K \rightarrow B \rightarrow N / M \rightarrow 0$ be any exact sequence. Then we have the following pullback diagram:

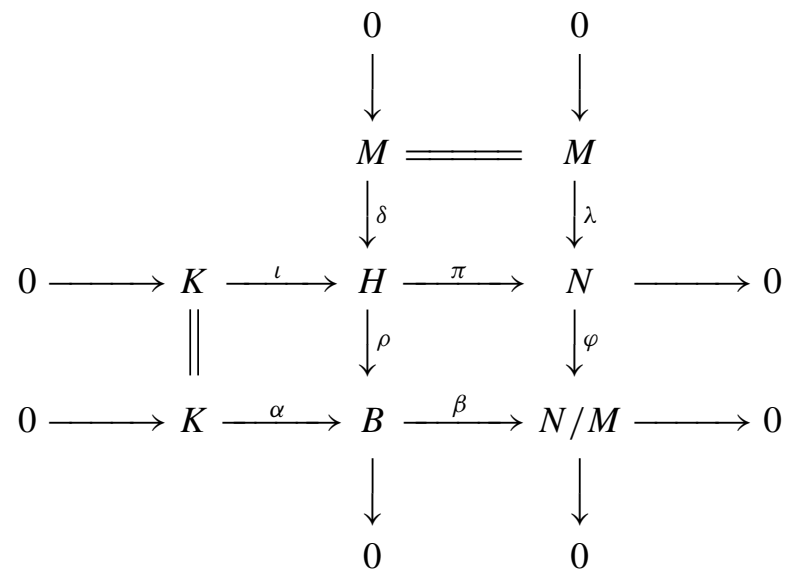

By (2), $H$ is $C P$-injective. So there exists $\gamma: N \rightarrow H$ such that $\delta=\gamma \lambda$. Note that $\lambda=\pi \delta=\pi \gamma \lambda$, thus $\pi \gamma$ is an isomorphism since $\lambda$ is an envelope. So $(\pi \gamma)^{-1} \lambda=\lambda$. It follows that

$$
\rho \gamma(\pi \gamma)^{-1}(M)=\rho \gamma(\pi \gamma)^{-1} \lambda(M)=\rho \gamma \lambda(M)=\rho \delta(M)=0 .
$$


Thus we get an induced map $\psi: N / M \rightarrow B$ such that $\psi \varphi=\rho \gamma(\pi \gamma)^{-1}$. Hence

$$
\beta \psi \varphi=\beta \rho \gamma(\pi \gamma)^{-1}=\varphi \pi \gamma(\pi \gamma)^{-1}=\varphi .
$$

So $\beta \psi=1$ since $\varphi$ is epic. Thus the sequence $0 \rightarrow K \rightarrow B \rightarrow N / M \rightarrow 0$ is split, and so $\operatorname{Ext}^{1}(N / M, K)=0$. By Theorem 2.9, $N / M$ is flat. Hence $N$ is flat.

(5) $\Rightarrow$ (2) If $0 \rightarrow A \rightarrow B \rightarrow C \rightarrow 0$ is an exact sequence of left $R$ modules, where $A$ is $C P$-injective and $C$ is a $C P$-injective envelope of a flat left $R$-module, then $C$ is flat by (5). So the sequence $0 \rightarrow A \rightarrow B \rightarrow C \rightarrow 0$ is split. Thus $B \cong A \oplus C$ is $C P$-injective.

$(1) \Rightarrow(6)$ follows from the fact that the cotorsion envelope of every flat left $R$-module is flat by Wakamatsu's Lemma.

$(6) \Rightarrow(1)$ is clear.

\section{Characterizations of $C P$-injective and $C P$-projective modules}

In [7], Divaani-Aazar, Esmkhani and Tousi have presented some criteria of $C P$-injective modules over a commutative ring $R$. In this section, we will give some other conditions that are equivalent to $C P$-injective ( $C P$-projective, $C P$-flat) modules.

THEOREM 4.1. Let $R$ be a ring. Then the following are equivalent for a left $R$-module $M$ :

(1) $M$ is a CP-injective left $R$-module.

(2) Every cyclically pure exact sequence $0 \rightarrow M \rightarrow N \rightarrow L \rightarrow 0$ of left $R$-modules is split.

(3) $M$ is injective relative to every cyclically pure exact sequence $0 \rightarrow A \rightarrow$ $B \rightarrow C \rightarrow 0$ of left $R$-modules with $B \mathscr{S}$-projective.

(4) $M$ is injective relative to every cyclically pure exact sequence $0 \rightarrow A \rightarrow$ $B \rightarrow C \rightarrow 0$ of left $R$-modules with $B C P$-projective.

Proof. (1) $\Rightarrow(2)$ and (1) $\Rightarrow(3) \Rightarrow$ (4) are obvious.

(2) $\Rightarrow(1)$ By [7, Theorem 2.5], there is a cyclically pure exact sequence $0 \rightarrow M \rightarrow N \rightarrow L \rightarrow 0$ with $N C P$-injective. So $M$ is $C P$-injective by (2).

(4) $\Rightarrow$ (1) Let $0 \rightarrow X \rightarrow Y \rightarrow Z \rightarrow 0$ be a cyclically pure exact sequence of left $R$-modules. By Lemma 2.6 (1), there is a cyclically pure exact sequence $0 \rightarrow U \rightarrow V \rightarrow Y \rightarrow 0$ with $V C P$-projective. Then we have the following 
pullback diagram:

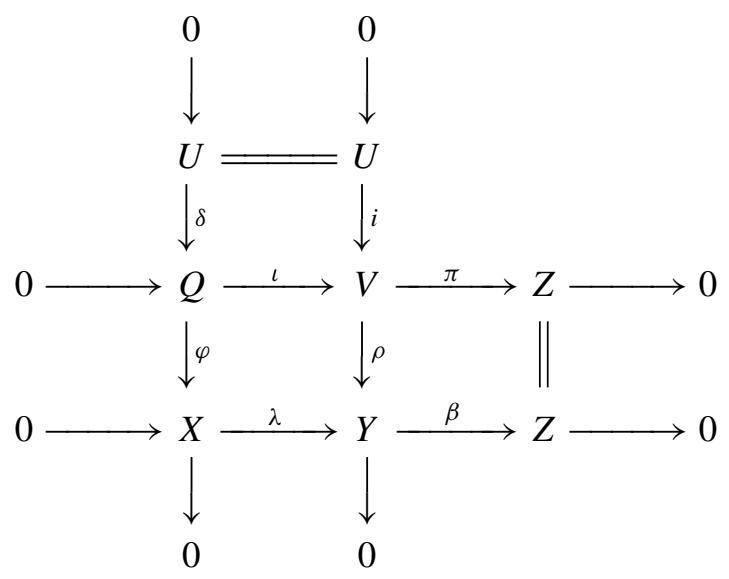

Thus $i=\iota \delta$ and $\pi=\beta \rho$. So $0 \rightarrow Q \rightarrow V \rightarrow Z \rightarrow 0$ is a cyclically pure exact sequence by Lemma 2.5. Let $\psi: X \rightarrow M$ be any homomorphism. By (4), there exists $\gamma: V \rightarrow M$ such that $\psi \varphi=\gamma \iota$. Since $\gamma \iota \delta=\psi \varphi \delta=0$, we have $\operatorname{ker}(\rho)=\operatorname{im}(i)=\operatorname{im}(\iota \delta) \subseteq \operatorname{ker}(\gamma)$. So there exists an induced map $\theta: Y \rightarrow M$ such that $\theta \rho=\gamma$. Thus $\psi \varphi=\theta \rho \iota=\theta \lambda \varphi$, and so $\psi=\theta \lambda$ since $\varphi$ is epic. Hence $M$ is $C P$-injective.

From Theorem 4.1, we deduce the following corollary.

COROLLARY 4.2. The following are equivalent for a right $R$-module $N$ :

(1) $\mathrm{N}$ is $\mathrm{CP}$-flat.

(2) For every cyclically pure exact sequence $0 \rightarrow A \rightarrow B \rightarrow C \rightarrow 0$ of left $R$-modules with $B C P$-projective, the sequence $0 \rightarrow N \otimes A \rightarrow$ $N \otimes B \rightarrow N \otimes C \rightarrow 0$ is exact.

Proof. (1) $\Rightarrow$ (2) is trivial.

(2) $\Rightarrow$ (1) Let $0 \rightarrow A \rightarrow B \rightarrow C \rightarrow 0$ be any cyclically pure exact sequence of left $R$-modules with $B C P$-projective. By (2), we get the exact sequence $0 \rightarrow N \otimes A \rightarrow N \otimes B \rightarrow N \otimes C \rightarrow 0$, which induces the exact sequence $0 \rightarrow \operatorname{Hom}\left(C, N^{+}\right) \rightarrow \operatorname{Hom}\left(B, N^{+}\right) \rightarrow \operatorname{Hom}\left(A, N^{+}\right) \rightarrow 0$. So $N^{+}$is $C P$-injective by Theorem 4.1. Thus $N$ is $C P$-flat by Lemma 2.3 (1).

Dual to Theorem 4.1, we have

THEOREM 4.3. The following are equivalent:

(1) $M$ is a $C P$-projective left $R$-module.

(2) Every cyclically pure exact sequence $0 \rightarrow D \rightarrow C \rightarrow M \rightarrow 0$ of left $R$-modules is split. 
(3) $M$ is projective relative to every cyclically pure exact sequence $0 \rightarrow$ $A \rightarrow B \rightarrow C \rightarrow 0$ of left $R$-modules with $B$ cotorsion.

(4) $M$ is projective relative to every cyclically pure exact sequence $0 \rightarrow$ $A \rightarrow B \rightarrow C \rightarrow 0$ of left $R$-modules with $B C P$-injective.

Proof. (1) $\Rightarrow(2)$ and (1) $\Rightarrow(3) \Rightarrow$ (4) are clear.

(2) $\Rightarrow$ (1) By Lemma 2.6 (1), there exists a cyclically pure exact sequence $0 \rightarrow D \rightarrow C \rightarrow M \rightarrow 0$ with $C C P$-projective. So $M$ is $C P$-projective by (2).

(4) $\Rightarrow$ (1) Let $0 \rightarrow K \rightarrow G \rightarrow H \rightarrow 0$ be a cyclically pure exact sequence of left $R$-modules. Suppose that $\lambda: G \rightarrow Q$ is a $C P$-injective envelope of $G$. Then we have the following pushout diagram:

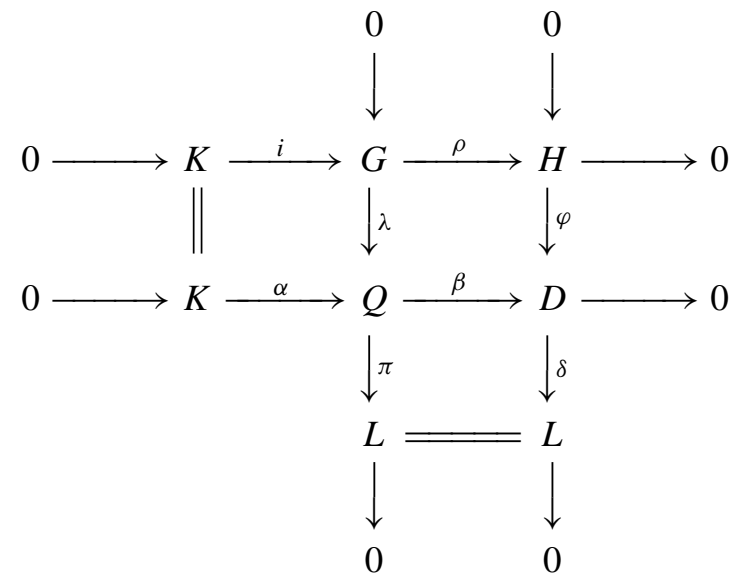

Thus $\pi=\delta \beta$ and $\alpha=\lambda i$, which implies that $0 \rightarrow K \rightarrow Q \rightarrow D \rightarrow 0$ is a cyclically pure exact sequence. Let $\psi: M \rightarrow H$ be any homomorphism. By (4), there exists $\gamma: M \rightarrow Q$ such that $\beta \gamma=\varphi \psi$. Since $\pi \gamma=\delta \beta \gamma=$ $\delta \varphi \psi=0, \operatorname{im}(\gamma) \subseteq \operatorname{ker}(\pi)=\operatorname{im}(\lambda)$. So we can define $\theta: M \rightarrow G$ by $\theta(x)=\lambda^{-1}(\gamma(x))$ for any $x \in M$. Thus $\varphi \psi=\beta \gamma=\beta \lambda \theta=\varphi \rho \theta$, and so $\psi=\rho \theta$ since $\varphi$ is monic. Hence $M$ is $C P$-projective.

As a consequence of Theorems 4.1 and 4.3, we have

COROLLARY 4.4. The following are equivalent for a ring $R$ :

(1) Every left $R$-module is $C P$-injective.

(2) Every left $R$-module is $C P$-projective.

(3) Every cyclically pure exact sequence $0 \rightarrow A \rightarrow B \rightarrow C \rightarrow 0$ of left $R$-modules is split. 
It is well known that any submodule of every projective left $R$-module is projective if and only if any quotient module of every injective left $R$-module is injective. The next theorem establishes an analogous result for the cyclically pure version.

THEOREM 4.5. Consider the following conditions for a ring $R$ :

(1) For any cyclically pure exact sequence $0 \rightarrow A \rightarrow B \rightarrow C \rightarrow 0$ of left $R$-modules with $B C P$-projective, $A$ is $C P$-projective.

(2) For any cyclically pure exact sequence $0 \rightarrow K \rightarrow M \rightarrow N \rightarrow 0$ of left $R$-modules with $M C P$-injective, $N$ is $C P$-injective.

(3) For any cyclically pure exact sequence $0 \rightarrow A \rightarrow B \rightarrow C \rightarrow 0$ of left $R$-modules with $B$ projective, $A$ is projective.

Then (1) $\Leftrightarrow(2) \Rightarrow(3)$. Moreover, if the class of CP-injective left $R$-modules is closed under extensions, then (3) $\Rightarrow$ (2).

Proof. (1) $\Rightarrow$ (2) Let $0 \rightarrow K \rightarrow M \stackrel{\pi}{\rightarrow} N \rightarrow 0$ be a cyclically pure exact sequence of left $R$-modules with $M C P$-injective and $0 \rightarrow A \stackrel{i}{\rightarrow} B \rightarrow C \rightarrow 0$ be a cyclically pure exact sequence of left $R$-modules with $B C P$-projective. For any $f: A \rightarrow N$, there exists $g: A \rightarrow M$ such that $\pi g=f$ since $A$ is $C P$-projective by (1). Hence there is $h: B \rightarrow M$ such that $h i=g$ since $M$ is $C P$-injective. It follows that $(\pi h) i=f$, and so $N$ is $C P$-injective by Theorem 4.1.

(2) $\Rightarrow$ (1) Let $0 \rightarrow A \stackrel{i}{\rightarrow} B \rightarrow C \rightarrow 0$ be a cyclically pure exact sequence of left $R$-modules with $B C P$-projective and $0 \rightarrow K \rightarrow M \stackrel{\pi}{\rightarrow} N \rightarrow 0$ be a cyclically pure exact sequence of left $R$-modules with $M C P$-injective. Then $N$ is $C P$-injective by (2). Thus for any $f: A \rightarrow N$, there exists $g: B \rightarrow N$ such that $f=g i$. It follows that there exists $h: B \rightarrow M$ such that $g=\pi h$ since $B$ is $C P$-projective. Hence $f=\pi(h i)$ and so $A$ is $C P$-projective by Theorem 4.3 .

(2) $\Rightarrow$ (3) Let $0 \rightarrow A \stackrel{i}{\rightarrow} B \rightarrow C \rightarrow 0$ be a cyclically pure exact sequence of left $R$-modules with $B$ projective. Then $C$ is flat by Theorem 2.9. Let $M$ be any left $R$-module. There is a cyclically pure sequence $0 \rightarrow M \rightarrow N \rightarrow$ $L \rightarrow 0$ with $N C P$-injective. By (2), $L$ is $C P$-injective. So we have the induced exact sequence

$$
0=\operatorname{Ext}^{1}(C, L) \longrightarrow \operatorname{Ext}^{2}(C, M) \longrightarrow \operatorname{Ext}^{2}(C, N)=0 .
$$

Thus $\operatorname{Ext}^{2}(C, M)=0$, and so we get the exact sequence

$$
0=\operatorname{Ext}^{1}(B, M) \longrightarrow \operatorname{Ext}^{1}(A, M) \longrightarrow \operatorname{Ext}^{2}(C, M)=0 .
$$

Therefore $\operatorname{Ext}^{1}(A, M)=0$, and so $A$ is projective. 
(3) $\Rightarrow$ (2) Let $0 \rightarrow K \rightarrow M \stackrel{\pi}{\rightarrow} N \rightarrow 0$ be a cyclically pure exact sequence of left $R$-modules with $M C P$-injective. Let $F$ be any flat left $R$-module. There exists a cyclically pure exact sequence $0 \rightarrow Q \rightarrow P \rightarrow F \rightarrow 0$ with $P$ projective. By (3), $Q$ is projective, so we have the induced exact sequence

$$
0=\operatorname{Ext}^{1}(Q, K) \longrightarrow \operatorname{Ext}^{2}(F, K) \longrightarrow \operatorname{Ext}^{2}(P, K)=0 .
$$

Thus $\operatorname{Ext}^{2}(F, K)=0$. So we have the induced exact sequence

$$
0=\operatorname{Ext}^{1}(F, M) \longrightarrow \operatorname{Ext}^{1}(F, N) \longrightarrow \operatorname{Ext}^{2}(F, K)=0 .
$$

Hence $\operatorname{Ext}^{1}(F, N)=0$, and so $N$ is cotorsion. By Proposition 3.5, $N$ is $C P$-injective.

Finally, we characterize $C P$-injective and $C P$-flat modules over a commutative ring.

THEOREM 4.6. Let $R$ be a commutative ring. The following are equivalent for an $R$-module $M$ :

(1) $M$ is a CP-injective R-module.

(2) $\operatorname{Hom}(F, M)$ is a $C P$-injective $R$-module for any $C P$-flat $R$-module $F$.

Proof. (1) $\Rightarrow$ (2) Let $0 \rightarrow A \rightarrow B \rightarrow C \rightarrow 0$ be a cyclically pure exact sequence of $R$-modules. For any ideal $I$ of $R$, we get the exact sequence

$$
0 \longrightarrow(R / I) \otimes A \longrightarrow(R / I) \otimes B \longrightarrow(R / I) \otimes C \longrightarrow 0 .
$$

Moreover, we claim that the exact sequence is also cyclically pure. In fact, let $J$ be an ideal of $R$. Since $(R / J) \otimes(R / I) \cong R /(J+I)$, the cyclically pure exact sequence $0 \rightarrow A \rightarrow B \rightarrow C \rightarrow 0$ induces the exactness of the sequence

$$
\begin{aligned}
0 \longrightarrow(R / J) \otimes((R / I) \otimes A) \longrightarrow & (R / J) \otimes((R / I) \otimes B) \\
& \longrightarrow(R / J) \otimes((R / I) \otimes C) \longrightarrow 0 .
\end{aligned}
$$

So the exact sequence $0 \rightarrow(R / I) \otimes A \rightarrow(R / I) \otimes B \rightarrow(R / I) \otimes C \rightarrow 0$ is cyclically pure. Thus, for any $C P$-flat $R$-module $F$, we get the exact sequence

$$
0 \longrightarrow F \otimes(R / I) \otimes A \longrightarrow F \otimes(R / I) \otimes B \longrightarrow F \otimes(R / I) \otimes C \longrightarrow 0 .
$$

Hence the sequence

$$
0 \longrightarrow(R / I) \otimes(F \otimes A) \longrightarrow(R / I) \otimes(F \otimes B) \longrightarrow(R / I) \otimes(F \otimes C) \longrightarrow 0
$$


is exact. So the exact sequence

$$
0 \longrightarrow F \otimes A \longrightarrow F \otimes B \longrightarrow F \otimes C \longrightarrow 0
$$

is cyclically pure. Since $M$ is $C P$-injective, we obtain the exact sequence

$0 \longrightarrow \operatorname{Hom}(F \otimes C, M) \longrightarrow \operatorname{Hom}(F \otimes B, M) \longrightarrow \operatorname{Hom}(F \otimes A, M) \longrightarrow 0$,

which gives rise to the exactness of the sequence

$$
\begin{aligned}
0 \longrightarrow \operatorname{Hom}(C, \operatorname{Hom}(F, M)) \longrightarrow & \operatorname{Hom}(B, \operatorname{Hom}(F, M)) \\
& \longrightarrow \operatorname{Hom}(A, \operatorname{Hom}(F, M)) \longrightarrow 0 .
\end{aligned}
$$

Thus $\operatorname{Hom}(F, M)$ is a $C P$-injective $R$-module.

(2) $\Rightarrow$ (1) is clear by letting $F=R$.

COROLlary 4.7. Let $R$ be a commutative ring. The following are equivalent for an $R$-module $N$ :

(1) $N$ is a $C P$-flat $R$-module.

(2) $\operatorname{Hom}(N, E)$ is a $C P$-injective $R$-module for any $C P$-injective $R$-module $E$.

(3) $N \otimes F$ is a $C P$-flat $R$-module for any $C P$-flat $R$-module $F$.

Proof. (1) $\Rightarrow$ (2) follows from Theorem 4.6.

(2) $\Rightarrow$ (3) Let $F$ be any $C P$-flat $R$-module. Then $F^{+}$is $C P$-injective by Lemma 2.3 (1). So $(N \otimes F)^{+} \cong \operatorname{Hom}\left(N, F^{+}\right)$is $C P$-injective by (2). Thus $N \otimes F$ is $C P$-flat.

(3) $\Rightarrow$ (1) is clear by letting $F=R$.

\section{REFERENCES}

1. Angeleri-Hügel, L., On Some Precovers and Preenvelopes, Habilitationsschrift, Univ. München 2000.

2. Bican, L., El Bashir, R., and Enochs, E. E., All modules have flat covers, Bull. London Math. Soc. 33 (2001), 385-390.

3. Brewer, J. W., and Costa, D. L., Contracted ideals and purity for ring extensions, Proc. Amer. Math. Soc. 53 (1975), 271-276.

4. Chase, S. U., Direct products of modules, Trans. Amer. Math. Soc. 97 (1960), 457-473.

5. Cohn, P. M., On the free product of associative rings, Math. Z. 71 (1959), 380-398.

6. Colby, R. R., Rings which have flat injective modules, J. Algebra 35 (1975), 239-252.

7. Divaani-Aazar, K., Esmkhani, M. A., and Tousi, M., Some criteria of cyclically pure injective modules, J. Algebra 304 (2006), 367-381.

8. Divaani-Aazar, K., Esmkhani, M. A., and Tousi, M., A criterion for rings which are locally valuation rings, Colloq. Math. 116 (2009), 153-164. 
9. Enochs, E. E., Injective and flat covers, envelopes and resolvents, Israel J. Math. 39 (1981), 189-209.

10. Enochs, E. E., Flat covers and flat cotorsion modules, Proc. Amer. Math. Soc. 92 (1984), 179-184.

11. Enochs, E. E., and Jenda, O. M. G., Relative Homological Algebra, de Gruyter Expos. Math. 30, de Gruyter, Berlin 2000.

12. Göbel, R., and Trlifaj, J.,Approximations and Endomorphism Algebras of Modules, de Gruyter Expos. Math. 41, de Gruyter, Berlin 2006.

13. Hochster, M., Cyclic purity versus purity in excellent Noetherian rings, Trans. Amer. Math. Soc. 231 (1977), 463-488.

14. Holm, H., and Jørgensen, P., Covers, precovers, and purity, Illinois J. Math. 52 (2008), 691-703.

15. Lam, T. Y., Lectures on Modules and Rings, Grad. Texts in Math. 189, Springer, New York 1999.

16. Mao, L. X., and Ding, N. Q., New characterizations of pseudo-coherent rings, Forum Math. 22 (2010), 993-1008.

17. Melkersson, L., Small cofinite irreducibles, J. Algebra 196 (1997), 630-645.

18. Prest, M., Model Theory and Modules, London Math. Soc. Lecture Note 130, Cambridge Univ. Press, Cambridge 1988.

19. Rada, J., and Saorin, M., Rings characterized by (pre)envelopes and (pre)covers of their modules, Comm. Algebra 26 (1998), 899-912.

20. Rothmaler, P., When are pure-injective envelopes of flat modules flat?, Comm. Algebra 30 (2002), 3077-3085.

21. Rotman, J. J., An Introduction to Homological Algebra, Pure Appl. Math. 85, Academic Press, New York 1979.

22. Stenström, B., Pure submodules, Ark. Mat. 7 (1967), 159-171.

23. Warfield Jr., R. B., Purity and algebraic compactness for modules, Pacific J. Math. 28 (1969), 699-719.

24. Wisbauer, R., Foundations of Module and Ring Theory, Gordon and Breach, Philadelphia 1991.

25. Xu, J., Flat Covers of Modules, Lecture Notes in Math. 1634, Springer, Berlin 1996.

INSTITUTE OF MATHEMATICS

NANJING INSTITUTE OF TECHNOLOGY

NANJING 211167

CHINA

E-mail: maolx2@hotmail.com 